\title{
Editorial
}

Manuel Burghardt*, Christian Wolff und Christa Womser-Hacker

\section{Informationsinfrastruktur und informationswissenschaftliche Methoden in den digitalen Geisteswissenschaften}

DOI 10.1515/iwp-2015-0052

Das vorliegende Themenheft setzt sich mit dem Verhältnis von Informationsinfrastruktur, den Digital Humanities und der Informationswissenschaft auseinander. Anders als in weiten Teilen der Natur-, Lebens- und Ingenieurwissenschaften hat sich für die Geisteswissenschaften nicht bereits seit den 1970er Jahren eine überregionale Informationsinfrastrukturlandschaft jenseits der großen (Staats-) Bibliotheken herausbilden können. ${ }^{1}$ Mit der Digitalisie-

1 In den 1970er Jahren sah das IuD-Programm flächendeckende Informationsinfrastrukturen vor, um durch fachbezogene Informationssysteme und -dienstleistungen verteiltes Wissen überregional an einer Stelle zu sammeln, zu sichern, zusammenzuführen und in angereicherter Form und bezogen auf ihre Nutzer wieder bereitzustellen. Im Laufe der Zeit bildete sich im Bereich der Fachinformation ein klarer Fokus auf den natur-, lebens- und ingenieurswissenschaftlichen Bereichen heraus, die ihren Nutzen und ihre Akzeptanz auch in monetärer Hinsicht beweisen konnten (z.B. Patentinformation, Rechtsdokumentation). Relativ unbemerkt und noch deutlich vor der Legitimationskrise der Geisteswissenschaften verschwand das in Saarbrücken geplante Fachinformationszentrum Geisteswissenschaften - zumindest institutionell - von der Bildfläche (Klein, 1987). Von den bis heute bestehenden Fachinformationszentren haben nur wenige geistes- und sozialwissenschaftliche Schwerpunkte (DIPF, Frankfurt am Main - Pädagogik / Bildungsforschung; GESIS, Köln Sozialwissenschaften; juris, Saarbrücken - Rechtswissenschaft).

*Kontaktperson: Dr. Manuel Burghardt, Institut für Information und Medien, Sprache und Kultur, Universität Regensburg, Lehrstuhl für Medieninformatik, 93040 Regensburg, Deutschland,

E-Mail:manuel.burghardt@ur.de,www.mi.ur.de/sekretariat-team/ manuel-burghardt/index.html, http://orcid.org/0000-0003-13549089

Prof. Dr. Christian Wolff, Institut für Information und Medien, Sprache und Kultur, Universität Regensburg, Lehrstuhl für Medieninformatik, 93040 Regensburg, Deutschland, E-Mail: christian.wolff@ur.de, www.medieninformatik.it, http://orcid.org/0000-0001-7278-8595 Prof. Dr. Christa Womser-Hacker, Institut für Informationswissenschaft und Sprachtechnologie, Universität Hildesheim, Universitätsplatz 1, 31141 Hildesheim, Deutschland, E-Mail: womser@uni-hildesheim.de,

www.uni-hildesheim.de/ womser rung, die alle Bereiche der Wissenschaft erfasst und nachhaltig verändert, stellt sich aber (auch) den Geisteswissenschaften die dringliche Frage, welche Dienstleistungen im Bereich der Informationsinfrastruktur benötigt werden, wer ihre Träger sein können und wie sich dabei der wissenschaftliche Prozess verändert. Die strukturellen und methodischen Herausforderungen gehen dabei weit über die traditionellen Probleme der Fachinformation im Bereich der Literaturdokumentation und -recherche hinaus und betreffen den gesamten wissenschaftlichen Arbeitsprozess und seine Kommunikationsmittel.

Die Informationswissenschaft ist prädestiniert dafür, in diesem Spannungsfeld eine tragende Rolle zu übernehmen, da sie seit ihrer Entstehung als Wissenschaft der Fachinformation bzw. der Informationsinfrastrukturen mit (allen) anderen Disziplinen zusammenarbeitet. Gleichzeitig versteht sie sich als diejenige Disziplin, die sich informationellen Prozessen und damit allen Aspekten im Umfeld von Informationsinfrastrukturen widmet. Dass sie dabei teilweise in Konkurrenz mit anderen Disziplinen wie den Digital Humanities - also den digitalen Geisteswissenschaften als Fach bzw. Disziplin - oder auch der Informatik steht, vermag nicht zu überraschen und hat bereits zu einer Vielzahl noch andauernder Abgrenzungsdebatten geführt.

Die nachfolgenden Artikel beleuchten die Beziehung zwischen Informationswissenschaft und Digital Humanities und gehen dabei sowohl auf Aspekte der Informationsinfrastruktur als auch auf methodische Schnittstellen zwischen diesen Bereichen ein:

Manuel Burghardt, Christian Wolff und Christa Womser-Hacker diskutieren einleitend das Verhältnis zwischen Informationswissenschaft und Digital Humanities. Dabei wird deutlich, dass sich beide Disziplinen nur unscharf definieren lassen, sich anhand der jeweiligen fachlichen Profile aber viele Anknüpfungspunkte und Parallelen zwischen Informationswissenschaft und digitalen Geisteswissenschaften auftun. Weiterhin geht der Artikel auf typische informationswissenschaftliche Forschungsbereiche ein und zeigt auf, welche fachlichen und methodischen Angebote sich daraus für die Geisteswissenschaften ergeben. 
Es folgt ein thematischer Block mit drei Beiträgen, welche die Informations- und Forschungsinfrastruktur in den Digital Humanities näher beschreiben. Den Anfang machen Gerhard Heyer, Thomas Eckart und Dirk Goldhahn, die am Beispiel der Initiative CLARIN (Common Language Resources and Technology Infrastructure) die Potenziale von IT-basierten Forschungsinfrastrukturen für die Geistesund Sozialwissenschaften aufzeigen. Dabei wird für einen konkreten Anwendungsfall illustriert, wie mithilfe der CLARIN-Infrastruktur Ressourcen gefunden, aufbereitet, analysiert und die Ergebnisse visualisiert werden können.

Mirjam Blümm, Stefan E. Funk und Sibylle Söring stellen mit DARIAH-DE (Digital Research Infrastructure for the Arts and Humanities) eine weitere Forschungsinfrastruktur für die Geisteswissenschaften vor. Der Beitrag geht zudem auf die virtuelle Forschungsumgebung TextGrid ein, die verschiedene Tools und Dienste für den gesamten geisteswissenschaftlichen Forschungsprozess sowie auch ein digitales Langzeitarchiv für digitale Texte und Quellen bereitstellt.

Schließlich präsentieren Manuel Burghardt und Christian Wolff in Zusammenarbeit mit den Ansprechpartnern des TCDH (Trier Center for Digital Humanities), des CCeH (Cologne Center for eHumanities) und des GCDH (Göttingen Centre for Digital Humanities) einen Überblick zum Leistungsspektrum der Zentren für Digital Humanities in Deutschland. Der Überblick bezieht sich im Wesentlichen auf die Bereiche „Struktur und Genese“, „Profil, Aufgaben und Dienste“, „Nutzer, Vernetzung und Projekte“ sowie „Ausblick und Strategie“.

Ein weiterer thematischer Block, bestehend aus drei Einzelbeiträgen, beschreibt Methoden und Projekte aus der Informationswissenschaft und Computerlinguistik, die konkrete Bezüge zu den Digital Humanities aufweisen. Den Anfang macht hier Anke Reinhold, die in ihrem Artikel die grundlegende informationswissenschaftliche Methode des Experteninterviews thematisiert. Sie erläutert dabei zunächst die Methode an sich und gibt darüber hinaus praktische Handlungsanweisungen zur Durchführung von Experteninterviews. Schließlich wird die Bedeutung der empirisch-qualitativen Datenanalyse für die Wissensmodellierung in den Digital Humanities diskutiert.

Ben Heuwing und Christa Womser-Hacker zeigen in ihrem Beitrag auf, wie informationswissenschaftliche Ansätze für die domänenspezifische, nutzerzentrierte Bedarfsanalyse in den Digital Humanities eingesetzt werden können. Dabei werden konkrete Erfahrungen aus dem laufenden Projekt „Welt der Kinder“ beschrieben, in dem ein interdisziplinäres Team aus Historikern, Computerlinguisten und Informationswissenschaftlern innovative Methoden entwickelt und evaluiert, mit denen ein großes histori- sches Schulbuchkorpus mit Mitteln der automatischen Textanalyse vergleichend ausgewertet wird.

Abschließend präsentieren Fritz Kliche und Ulrich Heid ein computerlinguistisches Tool für den einfachen und transparenten Zugang zur sprachtechnologischen Arbeit mit Textdaten. Die „Explorationswerkbank“, die im Rahmen des Verbundprojekts e-Identity für die politikwissenschaftliche Analyse unterschiedlicher Identitätskonzepte entwickelt wurde, ermöglicht u.a. die Segmentierung von Texten in strukturelle Einheiten sowie auch die Extraktion von konkreten Textobjekten. Die Autoren erläutern in ihrem Beitrag die wesentlichen Konzepte für einen einfachen Zugang und illustrieren potenzielle Anwendungsbereiche des Tools anhand von drei Fallbeispielen.

\section{Literatur}

Klein, J. (1987). Geisteswissenschaftliche Fachinformation in der Bundesrepublik Deutschland. Bibliothek 11 (3) (1987), 227-262.

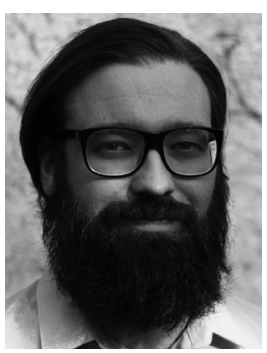

Dr. Manuel Burghardt

Institut für Information und Medien

Sprache und Kultur

Universität Regensburg

Lehrstuhl für Medieninformatik

93040 Regensburg

Deutschland

manuel.burghardt@ur.de

www.mi.ur.de/sekretariat-team/manuelburghardt/index.html

http://orcid.org/0000-0003-1354-9089

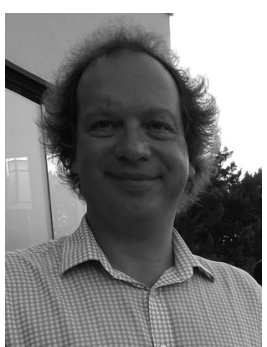

Prof. Dr. Christian Wolff

Institut für Information und Medien

Sprache und Kultur

Universität Regensburg

Lehrstuhl für Medieninformatik

93040 Regensburg

Deutschland

christian.wolff@ur.de

www.medieninformatik.it

http://orcid.org/0000-0001-7278-8595

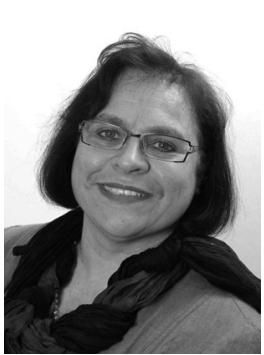

Prof. Dr. Christa Womser-Hacker

Institut für Informationswissenschaft und Sprachtechnologie

Universität Hildesheim

Universitätsplatz 1

31141 Hildesheim

Deutschland

womser@uni-hildesheim.de

www.uni-hildesheim.de/ womser 